\title{
Glycated albumin is a better indicator of the glucose excursion than predialysis glucose and hemoglobin A1C in hemodialysis patients
}

\author{
Yuki Tsuruta, Akiko Ichikawa, Kan Kikuchi, Yoshihisa Echida, Takako Onuki and Kosaku Nitta*
}

\begin{abstract}
Background: Glycated albumin (GA) has been reported to be a better indicator of glycemic control than hemoglobin A1c (HbA1c) in diabetic hemodialysis (HD) patients.

Methods: We performed a cross-sectional study to evaluate associations between daily glucose profiles based on blood glucose measurements at seven different times a day and their GA, HbA1c, and pre-dialysis glucose levels in $90 \mathrm{HD}$ patients with $(n=46)$ and without $(n=44)$ diabetes.

Results: The results of a univariate analysis showed that the GA, HbA1c, and pre-dialaysis glucose levels were significantly correlated with the daily glucose profiles. In a multivariate analysis, the GA levels were found to be independently correlated with maximum glucose levels $(F=9.448, P=0.0028)$ and mean amplitude of glucose excursion (MAGE, $F=5.169, P=0.0255$ ), but no significant correlation was found between the HbA1c levels and daily glucose profiles. The pre-dialysis glucose levels were independently correlated with minimum glucose levels ( $F=8.336, P=0.0049)$. In a stratified analysis, the identical trend was seen in the diabetic HD patients, but an independent correlation was found only between $\mathrm{HbA1c}$ values and mean glucose levels in nondiabetic HD patients.

Conclusions: GA levels may be a better indicator of glycemic control than $\mathrm{HbA1C}$ and pre-dialysis glucose levels, especially as a means of evaluating the glucose excursions in diabetic HD patients.
\end{abstract}

Keywords: Glycated albumin, Hemoglobin A1c, Hemodialysis, Glucose profiles

\section{Background}

Diabetes mellitus is the leading cause of chronic kidney disease and of being started on hemodialysis (HD) [1], and it is associated with cardiovascular disease that represents a common risk of morbidity and mortality in HD patients [2]. Hyperglycemia causes micro- and macroangiopathy in diabetic patients, and better glycemic control prevents diabetic complications [3, 4]. However, observational studies that have evaluated the relationship between glycemic control and the survival of HD patients with diabetes have yielded conflicting results [5-7]. Using serum glycated hemoglobin (HbA1c) to assess glycemic control in HD patients may result in underestimation of the level

\footnotetext{
* Correspondence: nitta@kc.twmu.ac.jp

Department of Medicine, Kidney Center, Tokyo Women's Medical University, 8-1 Kawada-cho, Shinjuku-ku, Tokyo 162-8666, Japan
}

of glycemic control, because the life span of the red blood cells of HD patients is shorter, because the percentage of immature red blood cells is increased by erythropoiesisstimulating agent therapy, and because blood loss may occur during HD therapy [8]. Previous studies have shown that glycated albumin (GA), whose levels are unaffected by the life-span of red blood cells, may be a better indicator of glycemic control in HD patients than HbA1c $[9,10]$. The Japanese Society for Dialysis Therapy recommends pre-dialysis glucose levels and GA levels as better indicators of glycemic control than $\mathrm{HbA1c}$ in diabetic HD patients [11]. However, these studies used only casual blood glucose levels as the gold standard for comparison with HbA1c and GA levels.

The glucose excursions have been shown to increase the risk of diabetic macroangiopathy [4, 12]. GA levels 
have been reported to be more useful indicator of glycemic control, especially as a means of evaluating postprandial glycemic excursion than HbA1c in diabetic patients without nephropathy [13]. The aim of this study was to investigate associations between the pre-dialysis glucose, HbA1c, GA levels of HD patients, and their daily glucose profiles.

\section{Methods}

\section{Subjects}

We performed a cross-sectional study of 90 HD patients who had been admitted to Shinjuku Ishikawa Hospital. Patients who had inflammatory disease, gastrointestinal hemorrhage, or malignant disease were excluded. Patients capable of an oral diet were included in this study, and they were mostly admitted because of vascular access trouble and social problem. The glucose-lowering therapy of the subjects had been unchanged for at least 3 months prior to enroll into this study.

After a full explanation of this study, written informed consent was obtained from each patient. The study was performed in accordance with the Declaration of Helsinki and the Principles of Good Clinical Practice, and the Institutional Research Ethics Committee approved the study protocol.

\section{Biochemical measurements}

All blood samples for biochemical measurements were collected before the first HD session after hospital admission. HbA1c, GA, and the other parameters were measured at reference laboratories (SRL Inc., Tokyo, Japan). The daily glucose profile was based on measurements made by medical staff before and $2 \mathrm{~h}$ after each meal and at bedtime, and the data was used to calculate the mean, minimum (Min), maximum (Max) glucose levels, and the mean amplitude of glucose excursion (MAGE) [14].

\section{Statistical analysis}

Continuous variables are reported as the mean \pm SD, and categorical variables are reported as percentages unless otherwise stated. The relationships among continuous variables were analyzed by the Pearson correlation test. A multiple linear regression analysis was performed to evaluate the relationships among glycated protein levels and various parameters of the daily glucose profile. $P$ values less than 0.05 were considered to indicate statistical significance. All analyses were performed with the JMP for Windows software program (SAS Institute, Cary, NC, USA).

\section{Results}

A total of $90 \mathrm{HD}$ patients were enrolled in this study. Their baseline characteristics, including laboratory data, are shown in Table 1 . The mean age of the subjects was $68.8 \pm 12.1$ years, and their mean HD vintage was 115.8 \pm 166.1 months. The patients who were started on HD therapy because of diabetic nephropathy accounted for $43.3 \%$, and patients diagnosed with type 2 diabetes accounted for $51.1 \%$. The baseline characteristics of the diabetic and nondiabetic HD patients are also shown in Table 1. Whereas the serum creatinine levels were significantly lower in the diabetic HD patients, the BMI and blood urea nitrogen levels tended to be higher but did not reach statistical significance. Oral hypoglycemic agents were being used to treat $28.3 \%$ of the diabetic HD patients, and $26 \%$ were on insulin therapy. Their glycemic parameters, including the Min, mean, Max glucose levels, Max-Min glucose levels, and MAGE values are shown in Table 2. All parameters, particularly the

Table 1 Baseline demographic data and clinical characteristics of the enrolled hemodialysis patients

\begin{tabular}{|c|c|c|c|c|}
\hline & All patients $(n=90)$ & Diabetic patients $(n=46)$ & Nondiabetic patients $(n=44)$ & $P$ value \\
\hline Age (years) & $68.8 \pm 12.1$ & $66.3 \pm 11.4$ & $71.3 \pm 12.5$ & 0.0536 \\
\hline Gender (male) & 66.6 & 73.9 & 59.1 & 0.135 \\
\hline Hemodialysis vintage (months) & $115.8 \pm 166.1$ & $102.4 \pm 194.9$ & $129.4 \pm 131.2$ & 0.447 \\
\hline Body mass index $\left(\mathrm{kg} / \mathrm{m}^{2}\right)$ & $21.2 \pm 3.9$ & $22.0 \pm 4.4$ & $20.4 \pm 3.1$ & 0.0596 \\
\hline Hemoglobin (g/dl) & $9.9 \pm 1.4$ & $10.2 \pm 1.4$ & $9.6 \pm 1.5$ & 0.062 \\
\hline Albumin $(\mathrm{g} / \mathrm{dl})$ & $3.3 \pm 0.4$ & $3.3 \pm 0.4$ & $3.3 \pm 0.4$ & 0.7119 \\
\hline Blood urea nitrogen (mg/dl) & $60.1 \pm 17.8$ & $62.1 \pm 18.7$ & $58.0 \pm 16.8$ & 0.2767 \\
\hline Creatinine (mg/dl) & $9.7 \pm 3.1$ & $9.1 \pm 3.0$ & $10.4 \pm 3.2$ & 0.0480 \\
\hline Diabetes (\%) & 51.1 & 100 & 0 & $<0.0001$ \\
\hline Oral hypoglycemic agent (\%) & 14.4 & 28.3 & 0 & $<0.0001$ \\
\hline Insulin (\%) & 13.3 & 26.1 & 0 & $<0.0001$ \\
\hline Erythropoiesis-stimulating agents (IU/week) & $5270.8 \pm 4186.5$ & $5225.5 \pm 4428.9$ & $5318.2 \pm 3968.0$ & 0.9171 \\
\hline
\end{tabular}

Variables are presented as mean \pm SD

${ }^{\mathrm{a}}$ Diabetic patients vs nondiabetic patients 
Table 2 Glycemic control parameters among the enrolled hemodialysis patients

\begin{tabular}{lllll}
\hline & All patients $(n=90)$ & Diabetic patients $(n=46)$ & Nondiabetic patients $(n=44) \quad$ value \\
\hline Min glucose $(\mathrm{mg} / \mathrm{dl})$ & $109.1 \pm 31.3$ & $120.5 \pm 34.7$ & $97.2 \pm 22.0$ & $<0.001$ \\
Mean glucose $(\mathrm{mg} / \mathrm{dl})$ & $152.3 \pm 38.6$ & $175.5 \pm 38.6$ & $128.0 \pm 18.2$ & $<0.001$ \\
Max glucose $(\mathrm{mg} / \mathrm{dl})$ & $203.4 \pm 62.0$ & $241.9 \pm 61.2$ & $163.2 \pm 28.3$ & $<0.001$ \\
Max-Min glucose $(\mathrm{mg} / \mathrm{dl})$ & $100.3 \pm 58.9$ & $130.6 \pm 64.9$ & $68.7 \pm 27.8$ & $<0.001$ \\
MAGE & $66.8 \pm 38.3$ & $85.4 \pm 42.1$ & $47.4 \pm 20.7$ & $<0.001$ \\
Pre-dialysis glucose $(\mathrm{mg} / \mathrm{dl})$ & $144.6 \pm 49.6$ & $165.2 \pm 56.3$ & $123.2 \pm 29.1$ & $<0.001$ \\
HbA1c $(\%)$ & $5.4 \pm 0.9$ & $5.8 \pm 1.1$ & $4.9 \pm 0.5$ & $<0.001$ \\
GA (\%) & $19.1 \pm 4.7$ & $21.9 \pm 4.7$ & $16.1 \pm 2.4$ & $<0.001$ \\
\hline
\end{tabular}

Variables are presented as mean \pm SD

Min minimum, Max maximum, MAGE mean amplitude of glucose excursion, HbA1c hemoglobin A1c, GA glycated albumin

${ }^{a}$ Diabetic patients vs nondiabetic patients

Max glucose levels and MAGE values, were higher in the diabetic HD patients. The pre-dialysis glucose levels were close with the mean glucose levels.

\section{Glycated albumin, $\mathrm{HbA1c}$, and pre-dialysis glucose levels in all patients}

The GA values, HbA1c values, and pre-dialysis glucose levels of the subjects as a whole were correlated with their daily glucose profiles (Table 3 ).

The GA levels, HbA1c levels, and pre-dialysis glucose levels of the subjects as a whole were correlated with their daily glucose profiles (Table 3). In univariate analysis, the HbA1c values were strongly correlated with the mean glucose levels as a whole. The pre-dialysis glucose levels were also significantly correlated with the Min and mean glucose levels. The GA values, on the other hand, were strongly correlated with the Max glucose levels as well as with the mean glucose levels.

Accordingly to the multivariate analysis, the GA values were independently correlated with the Max glucose levels and MAGE values, and the strongest correlation was found between the GA values and Max glucose levels, but a significant correlation was not found between the HbAlc values and daily glucose profiles. The pre-dialysis glucose levels were independently correlated with the Min glucose levels.

Table 3 Association between the indicators of glycemic control and parameters of daily glucose profiles in hemodialysis patients with and without diabetes

\begin{tabular}{|c|c|c|c|c|c|c|c|c|c|c|c|c|c|}
\hline \multirow{3}{*}{ Factors } & \multirow{3}{*}{ Parameters } & \multicolumn{4}{|c|}{ All patients $(n=90)$} & \multicolumn{4}{|c|}{ Diabetic patients $(n=46)$} & \multicolumn{4}{|c|}{ Non diabetic patients $(n=44)$} \\
\hline & & \multicolumn{2}{|c|}{ Univariate } & \multicolumn{2}{|c|}{ Multivariate } & \multicolumn{2}{|c|}{ Univariate } & \multicolumn{2}{|c|}{ Multivariate } & \multicolumn{2}{|c|}{ Univariate } & \multicolumn{2}{|c|}{ Multivariate } \\
\hline & & $\mathrm{R} 2$ & $P$ & $\mathrm{~F}$ & $P$ & $\mathrm{R} 2$ & $P$ & $\mathrm{~F}$ & $P$ & $\mathrm{R} 2$ & $P$ & $\mathrm{~F}$ & $P$ \\
\hline \multirow[t]{5}{*}{$\overline{\mathrm{GA}}(\%)$} & Min glucose $(\mathrm{mg} / \mathrm{dl})$ & 0.216 & $<0.0001$ & 0.917 & 0.341 & 0.174 & 0.004 & 1.167 & 0.2863 & 0.000 & 0.941 & 0.459 & 0.502 \\
\hline & Mean glucose (mg/dl) & 0.532 & $<0.0001$ & 2.665 & 0.1063 & 0.385 & $<0.0001$ & 1.334 & 0.2548 & 0.098 & 0.0383 & 0.848 & 0.3627 \\
\hline & Max glucose (mg/dl) & 0.511 & $<0.0001$ & 9.448 & 0.0028 & 0.329 & $<0.0001$ & 7.906 & 0.0075 & 0.122 & 0.0201 & 0.012 & 0.9152 \\
\hline & MAGE & 0.286 & $<0.0001$ & 5.169 & 0.0255 & 0.111 & 0.0235 & 6.349 & 0.0157 & 0.114 & 0.0253 & 0.821 & 0.3705 \\
\hline & Max-Min glucose (mg/dl) & 0.323 & $<0.0001$ & & & 0.136 & 0.0118 & & & 0.124 & 0.0192 & & \\
\hline \multirow[t]{5}{*}{$\mathrm{HbA1c}(\%)$} & Min glucose (mg/dl) & 0.363 & $<0.0001$ & 2.726 & 0.1024 & 0.385 & $<0.0001$ & 1.849 & 0.1814 & 0.027 & 0.2861 & 0.0018 & 0.9664 \\
\hline & Mean glucose (mg/dl) & 0.501 & $<0.0001$ & 3.751 & 0.0561 & 0.363 & $<0.0001$ & 0.636 & 0.4299 & 0.274 & 0.0003 & 8.259 & 0.0066 \\
\hline & Max glucose (mg/dl) & 0.393 & $<0.0001$ & 2.957 & 0.0892 & 0.226 & 0.0008 & 2.995 & 0.0910 & 0.181 & 0.0040 & 1.898 & 0.1761 \\
\hline & MAGE & 0.171 & $<0.0001$ & 2.817 & 0.0970 & 0.038 & 0.1918 & 2.959 & 0.0930 & 0.122 & 0.0200 & 2.889 & 0.0972 \\
\hline & Max-Min glucose (mg/dl) & 0.169 & $<0.0001$ & & & 0.032 & 0.2334 & & & 0.118 & 0.0223 & & \\
\hline \multirow[t]{5}{*}{ Pre-dialysis glucose (mg/dl) } & Min glucose (mg/dl) & 0.363 & $<0.0001$ & 8.336 & 0.0049 & 0.435 & $<0.0001$ & 9.730 & 0.0033 & 0.014 & 0.4449 & 0.043 & 0.8366 \\
\hline & Mean glucose (mg/dl) & 0.376 & $<0.0001$ & 0.950 & 0.3324 & 0.262 & 0.0003 & 0.012 & 0.9150 & 0.168 & 0.0058 & 1.198 & 0.2804 \\
\hline & Max glucose (mg/dl) & 0.312 & $<0.0001$ & 0.802 & 0.3729 & 0.167 & 0.0049 & 0.139 & 0.7114 & 0.182 & 0.0039 & 0.001 & 0.9724 \\
\hline & MAGE & 0.147 & 0.0002 & 0.259 & 0.6124 & 0.034 & 0.2190 & 0.009 & 0.9266 & 0.127 & 0.0174 & 0.531 & 0.4705 \\
\hline & Max-Min glucose (mg/dl) & 0.142 & 0.0003 & & & 0.027 & 0.2773 & & & 0.132 & 0.0153 & & \\
\hline
\end{tabular}




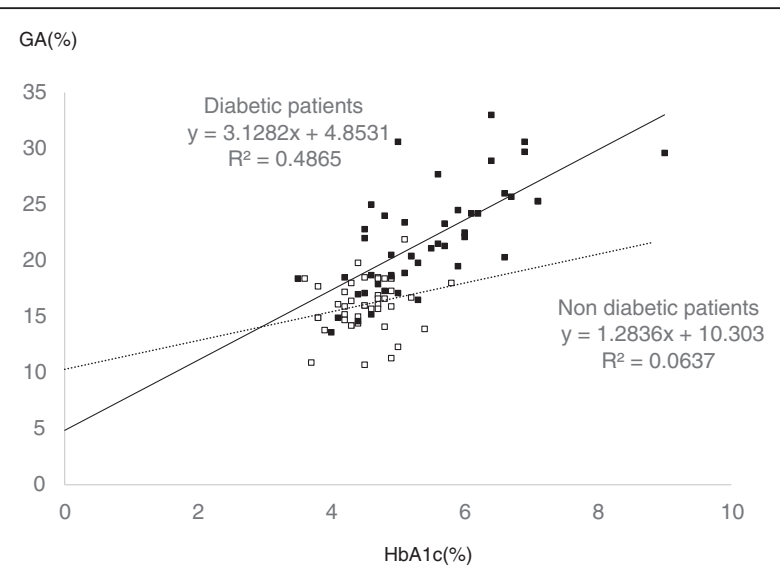

Fig. 1 Correlation between the glycated albumin (GA) and hemoglobin $\mathrm{A} 1 \mathrm{c}(\mathrm{HbA} 1 \mathrm{c})$ levels of diabetic (black squares) and nondiabetic (white squares) hemodialysis patients

\section{Diabetic patients and nondiabetic patients}

As shown in Fig. 1, the GA values of the diabetic HD patients were correlated with their HbA1c values $(r=0.697$, $P<0.0001)$, but not of the nondiabetic HD patients $(r=0.252, P=0.0984)$. The $\mathrm{GA} / \mathrm{HbA} 1 \mathrm{c}$ ratio was 3.1 in the diabetic HD patients.

The relationship of each indicator of glucose control and the Min, mean, Max glucose levels, and MAGE values of diabetic and nondiabetic $\mathrm{HD}$ patients is also shown in Table 3. The GA values, HbA1c values, and pre-dialysis glucose levels were correlated with the daily glucose profile in diabetic patients similar in all subjects. The identical trend was seen in multivariate analysis that the independently correlation was found in the GA values with Max glucose levels and MAGE values, which show the glucose excursion, and pre-dialysis glucose levels with the Min glucose levels. In contrast, the correlation of daily glucose profiles and GA values was weaker than the correlation between the HbA1c values and pre-dialysis glucose levels in nondiabetic HD patients. The results of the multivariate analysis did not show any independent correlation between the GA values, pre-dialysis glucose levels, and their daily glucose profiles in nondiabetic HD patients. On the other hand, the HbA1c values were independently correlated with the mean glucose levels in nondiabetic HD patients.

\section{Discussion}

The results of this study showed significant correlations between the pre-dialysis glucose, HbA1c values, and GA values and their daily glucose profiles in HD patients. They also showed that only the GA levels were independently correlated with the glucose excursions.

Since the lifespan of erythrocytes is approximately 120 days, serum HbA1c levels reflect blood glucose levels over the preceding several months. GA levels are used as an indicator of short-term glycemic control, because metabolic albumin turnover is faster than hemoglobin turnover; albumin has a lifespan of approximately 17 to 23 days. Furthermore, large blood glucose fluctuation could enhance the glycation and oxidation reaction of albumin, followed by increases in GA levels. Moreover, hyperglycemia has been reported to reduce erythrocyte survival [15]. Since diabetic HD patients have many factors that affect their serum HbA1c levels, their GA levels were better indicator of glycemic control, especially of the glucose excursions than HbA1c in HD patients who have a large glucose fluctuation. We did not find a correlation between hemoglobin, ESA use and HbA1c, serum albumin and GA that have been reported by previous studies $[9,10]$. However, the GA/HbA1c ratio in the present study was higher than reported in a previous study [13].

A large cohort study of 1484 HD patients reported finding that poor glycemic control of maintenance HD patients was not associated with their mortality [7]. This study used casual glucose and HbA1c levels as the indicator of glycemic control, and this cohort included nondiabetic HD patients, and their HbA1c levels were generally low. The GA, HbA1c, and pre-dialysis glucose levels in our study were not correlated with their glucose excursions of nondiabetic HD patients. One of the potential reasons for this finding is that the glucose levels in nondiabetic HD patients fluctuated within a narrow range. The results of other studies that investigated the impact of HbA1c on the survival of $\mathrm{HD}$ patients showed only extremely high or low HbA1c levels were associated with longer survival $[5,6]$. Thus, the optimal target glycemic control to improve the survival of diabetic HD patients is still unknown [16]. It is well known that postprandial hyperglycemia in diabetic patients is associated with the progression of diabetic macroangiopathy $[17,18]$. Our data showed that GA levels were a better indicator of the glucose fluctuations. A few recent studies have reported finding that high GA levels were associated with increased mortality in diabetic HD patients [19, 20].

Our study had several limitations. First, only hospitalized patients were enrolled as subjects. The hospital diet provided 30-35 kcal/ $\mathrm{kg} /$ day, and since it may have differed from the subjects' usual diet, the difference may have affected their daily glycemic profiles. Second, the daily glucose profiles were based on a seven-point blood glucose measurement a day, and a continuous glucosemonitoring system, which provides continuous detailed records of glucose levels, may have provided more accurate information.

\section{Conclusions}

HbA1c and pre-dialysis glucose levels were affected by the daily glucose profiles in diabetic HD patients. The 
GA levels could be a better indicator of glycemic control than $\mathrm{HbA1c}$ and pre-dialysis glucose levels, because serum GA levels may reflect the glucose excursion. These results warrant further trials to assess the usefulness of GA levels as an indicator of diabetic complications and mortality in diabetic HD patients.

\section{Competing interests}

The authors declare that they have no competing interests.

\section{Authors' contributions}

YT planned the study, searched the literature, assessed the studies, extracted the data, analyzed the data, and prepared the article. KK searched the literature, assessed the studies, and assisted in the article preparation. Al and YE assisted in the data analysis. TO searched the literature and assisted in the article preparation. KN planned the study, analyzed the data, and assisted in the article preparation. All authors read and approved the final manuscript.

Received: 17 July 2015 Accepted: 13 September 2015

Published online: 26 January 2016

\section{References}

1. Mehdi U, Toto RD. Anemia, diabetes, and chronic kidney disease. Diabetes Care. 2009:32:1320-6.

2. Nakai S, Hanafusa N, Masakane I, Taniguchi M, Hamano T, Shoji T, et al. An overview of regular dialysis treatment in Japan (as of 31 December 2012). Ther Apher Dial. 2014;18:535-602.

3. The Diabetes Control and Complications Trial Research Group. The relationship of glycemic exposure $(\mathrm{HbA} 1 \mathrm{c})$ to the risk of development and progression of retinopathy in the diabetes control and complications trial. Diabetes. 1995;44:968-83.

4. Hanefeld M, Fischer S, Julius U, Schulze J, Schwanebeck U, Schmechel H, et al. Risk factors for myocardial infarction and death in newly detected NIDDM: the Diabetes Intervention Study, 11-year follow-up. Diabetologia. 1996:39:1577-83.

5. Kalantar-Zadeh K, Kopple JD, Regidor DL, Jing J, Shinaberger CS, Aronovitz J, et al. A1C and survival in maintenance hemodialysis patients. Diabetes Care. 2007;30:1049-55.

6. Williams ME, Lacson Jr E, Teng M, Ofsthun N, Lazarus JM. Hemodialyzed type I and type II diabetic patients in the US: characteristics, glycemic control, and survival. Kidney Int. 2006;70:1503-9.

7. Shurraw S, Majumdar SR, Thadhani R, Wiebe N, Tonelli M, Alberta Kidney Disease Network. Glycemic control and the risk of death in 1,484 patients receiving maintenance hemodialysis. Am J Kidney Dis. 2010;55:875-84.

8. Nakao T, Matsumoto H, Okada T, Han M, Hidaka H, Yoshino M, et al. Influence of erythropoietin treatment on hemoglobin A1c levels in patients with chronic renal failure on hemodialysis. Intern Med. 1998;37:826-30.

9. Inaba M, Okuno S, Kumeda Y, Yamada S, Imanishi Y, Tabata T, et al. Glycated albumin is a better glycemic indicator than glycated hemoglobin values in hemodialysis patients with diabetes: effect of anemia and erythropoietin injection. J Am Soc Nephrol. 2007;18:896-903.

10. Peacock TP, Shihabi ZK, Bleyer AJ, Dolbare EL, Byers JR, Knovich MA, et al. Comparison of glycated albumin and hemoglobin $A(1 \mathrm{c})$ levels in diabetic subjects on hemodialysis. Kidney Int. 2008;73:1062-8.

11. Nakao T, Inaba M, Abe M, Kaizu K, Shima K, Babazono T, et al. Best practice for diabetic patients on hemodialysis 2012. Ther Apher Dial. 2015;19 Suppl 1:40-66.

12. Hanefeld M, Cagatay M, Petrowitsch T, Neuser D, Petzinna D, Rupp M. Acarbose reduces the risk for myocardial infarction in type 2 diabetic patients: meta-analysis of seven long-term studies. Eur Heart J. 2004;25:10-6.

13. Yoshiuchi K, Matsuhisa M, Katakami N, Nakatani Y, Sakamoto K, Matsuoka T, et al. Glycated albumin is a better indicator for glucose excursion than glycated hemoglobin in type 1 and type 2 diabetes. Endocr J. 2008:55:503-7.

14. Service FJ, Molnar GD, Rosevear JW, Ackerman E, Gatewood LC, Taylor WF. Mean amplitude of glycemic excursions, a measure of diabetic instability. Diabetes. 1970;19:644-55.

15. Virtue MA, Furne JK, Nuttall FQ, Levitt MD. Relationship between GHb concentration and erythrocyte survival determined from breath carbon monoxide concentration. Diabetes Care. 2004;27:931-5.

16. Ix JH. Hemoglobin A1C in hemodialysis patients: should one size fit all? Clin J Am Soc Nephrol. 2010;5:1539-41.
17. The DECODE Study Group, the European Diabetes Epidemiology Group. Glucose tolerance and mortality: comparison of WHO and American Diabetes Association diagnostic criteria. Lancet. 1999;354:617-21.

18. Bonora E, Muggeo M. Postprandial blood glucose as a risk factor for cardiovascular disease in type II diabetes: the epidemiological evidence. Diabetologia. 2001;44:2107-14

19. Fukuoka K, Nakao K, Morimoto H, Nakao A, Takatori Y, Arimoto K, et al. Glycated albumin levels predict long-term survival in diabetic patients undergoing haemodialysis. Nephrology (Carlton). 2008;13:278-83.

20. Isshiki K, Nishio T, Isono M, Makiishi T, Shikano T, Tomita K, et al. Glycated albumin predicts the risk of mortality in type 2 diabetic patients on hemodialysis: evaluation of a target level for improving survival. Ther Apher Dial. 2014;18:434-42.

\section{Submit your next manuscript to BioMed Central and take full advantage of:}

- Convenient online submission

- Thorough peer review

- No space constraints or color figure charges

- Immediate publication on acceptance

- Inclusion in PubMed, CAS, Scopus and Google Scholar

- Research which is freely available for redistribution

Submit your manuscript at www.biomedcentral.com/submit 